\title{
Inventário de Fontes de Estresse Acadêmico no Curso de Medicina (IFSAM)
}

\author{
An Inventory of Sources of Stress During \\ Medical Education (IASSME)
}

Elizabete Maria Ferraz Loureiro ${ }^{I}$ Teresa Mendonça McIntyre ${ }^{I I}$ Rui Mota-Cardoso ${ }^{I}$ Maria Amélia Ferreira ${ }^{I}$

\section{PALAVRAS-CHAVE}

- Estresse.

- Estudantes de medicina.

- Educação médica.

- Esgotamento profissional.

- Psicometria.
Recebido em: 26/11/2007

Reencaminhado em: 21/01/2008

Reencaminhado em: 16/03/2008

Aprovado em: 12/07/2008
REVISTA BRASILEIRA DE EDUCAÇÃO MÉDICA
${ }^{I}$ Universidade do Porto, Porto, Portugal.

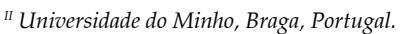

O Inventário de Fontes de Estresse Acadêmico no Curso de Medicina (IFSAM) foi desenvolvido para caracterizar as principais fontes de estresse acadêmico dos estudantes da Faculdade de Medicina da Universidade do Porto (FMUP) e a intensidade com que são experienciadas. O inventário (31 itens) foi construído por meio de entrevistas semiestruturadas a estudantes $(n=80)$ dos seis anos da licenciatura. Suas propriedades psicométricas foram testadas num estudo com 251 estudantes dos seis anos do curso (160 do sexo feminino e 91 do sexo masculino). A validade de constructo foi avaliada mediante análise fatorial que resultou numa solução de cinco fatores ("exigências do curso", "exigências humanas", "estilos de vida", "competição" e "adaptação") com 54,8\% da variância explicada e boa representatividade do constructo. O IFSAM total evidencia ainda uma boa fidelidade, com um coeficiente de consistência interna de.88. Os resultados revelam que o IFSAM apresenta boas características psicométricas na amostra, podendo constituir um instrumento útil na avaliação das fontes e intensidade de estresse acadêmico do curso de Medicina noutras instituições.

\section{ABSTRACT}

The Inventory of Academic Sources of Stress in Medical Education (IASSME) was developed to characterize the main sources of academic stress for students at the School of Medicine, University of Porto (FMUP), as well as the intensity with which they experience such stress. The 31-item inventory resulted from semi-structured interviews with students $(n=80)$ from all six years of medical school. The inventory's psychometric properties were tested on 251 students from the six years (160 females and 91 males). Construct validity was assessed by means of factor analysis, resulting in a five-factor solution ("course demands", "human demands", "lifestyle", "academic competition", and "academic adjustment"), with a total of $54.8 \%$ of the total variance explained as well as good representation of the construct. The total inventory showed good reliability and internal consistency of 0.88. Based on the findings, the IASSME displayed good psychometric characteristics in this sample and can be a useful instrument for assessing sources of academic stress and their intensity in other medical schools. 


\section{INTRODUÇÃO}

Os estudantes de Medicina estão expostos a numerosos estressores $^{1,2,3}$. Por um lado, é o curso que, nomeadamente em Portugal, exige a classificação de acesso mais elevada. Neste contexto, quando iniciam o curso, os estudantes o fazem após um ano particularmente desgastante. Por outro lado, passam vários anos e muitas horas a estudar para exames que determinarão seu futuro (ocupação). É que, como diz Adams ${ }^{3}$, o currículo médico não é simples, e o estresse experienciado na tentativa de compreender os conteúdos complexos pode ser intenso. De fato, a progressão no curso e na profissão médica exige sacrifícios emocionais e pessoais. Por um lado, as faculdades de Medicina possibilitam a aquisição de competências necessárias ao (bom) exercício do ofício, mas também são o local onde se adquirem os hábitos que tornam estes estudantes e futuros profissionais susceptíveis ao burnout ${ }^{4}$.

Quanto às fontes de estresse nos estudantes de Medicina, vários estudos têm comprovado que as maiores preocupações dos estudantes estão relacionadas com a área acadêmica, em particular com a sobrecarga de trabalho, exigências e desempenho $^{4,9}$. Existe ainda consenso generalizado de que, relativamente à análise da pontuação total dos estressores, são os estudantes do sexo feminino que os percepcionam de forma mais intensa. Também se salientam a falta de tempo e dedicação exigida, a ansiedade em torno da qualidade e dificuldade do material a estudar e o sistema de avaliação ${ }^{5}$. Embora os currículos tenham sofrido alterações em termos de aproximação da clínica desde os primeiros anos do curso, como refere $\mathrm{Knight}^{6}$, o colidir de expectativas pode ser muito forte para estudantes que, preservando o ideal de "curar" doentes na fase inicial do curso, têm de se conformar com um currículo de ciências básicas bastante intenso. No mesmo contexto, Miller ${ }^{7}$, após investigação com estudantes do primeiro ano, postulou que estes se sentiam estressados com a natureza e a sobrecarga de trabalho envolvidas no curso de Medicina, bem como com a estrutura do próprio curso e métodos de ensino. Afirmou, ainda, que os mesmos admitiam dificuldades na gestão de tempo, particularmente no que concerne ao equilíbrio entre as atividades sociais e as tarefas acadêmicas. Wolf et al. ${ }^{8}$ relataram, por sua vez, que estudantes do primeiro ano de Medicina se queixavam da limitação de oportunidades para o desenvolvimento de relações pessoais. $\mathrm{O}$ estudo evidenciou também que no final do ano os estudantes apresentavam mais sintomas depressivos, atingindo a incidência máxima no final do segundo ano.

$\mathrm{Na}$ opinião de Shaw et $\mathrm{al}^{9}$, e Toews et $\mathrm{al}^{10}$, os estressores específicos dos anos básicos se resumem às pressões com $o$ tempo, ao sistema de avaliação, a problemas financeiros (devido ao tempo e custo do curso de Medicina) e à competição por um bom desempenho; são de salientar, ainda, o tédio que advém da rotina de memorização de informação, a solidão, o medo do insucesso e a dependência prolongada dos pais. Por outro lado, no âmbito dos anos clínicos, evidenciam-se os conflitos interpessoais com colegas, o receio do aumento de responsabilidades, a solidão, o confronto com o sofrimento, o desconforto sentido na realização de exames físicos e a discussão de assuntos pessoais e sexuais com os pacientes. Os mesmos autores defendem, também, que os níveis de estresse se revelam elevados no início do primeiro ano e antes do período de avaliação, e baixos no final do quarto ano.

De acordo com Coles ${ }^{11}$, quatro aspectos merecem destaque quando nos reportamos ao estresse na educação médica. Em primeiro lugar, o autor salienta o currículo como fonte de estresse, uma vez que é constituído por uma sobrecarga de informações que muitas vezes se apresentam pouco relevantes para a prática. De fato, com frequência se constatam ligações tênues entre os conhecimentos teóricos dos cursos básicos e as experiências clínicas. Em segundo lugar, refere o papel dos professores, adiantando que muitos dos medical teachers nunca receberam formação pedagógica, o que afeta o modo como os estudantes aprendem e as relações de suporte que são construídas. Em terceiro lugar, o autor constata que o clima da educação médica é frequentemente pouco apoiador e ameaçador, quando, na realidade, deveria ser colaborador. Por último, em quarto lugar, refere que muitas escolas médicas ainda não dispõem de serviços de apoio aos estudantes e professores, de forma a prevenir efeitos nocivos do estresse ou permitir lidar de modo satisfatório com eles, uma vez desenvolvidos.

\section{OBJETIVOS DO ESTUDO}

Os autores que teorizam sobre estresse o entendem de três modos: (i) o estressor/fontes de estresse, isto é, o que desencadeia a resposta de estresse; (ii) a resposta de estresse, que pode ser física, psicológica ou social; e (iii) o processo de estresse, isto é, o resultado da interação do indivíduo com o estressor ${ }^{12}$. No estudo do estresse acadêmico, é importante avaliar todos estes aspectos. Já mencionamos que outros autores têm identificado vários estressores na experiência acadêmica dos estudantes de Medicina. No entanto, esta experiência poderá variar de curso para curso, com a instituição e de cultura para cultura. Assim, por exemplo, os estressores dos estudantes que frequentam cursos de Medicina nos EUA não se adaptam em sua totalidade aos estudantes portugueses, pois vários fatores se apresentam claramente diferentes, como aspectos relacionados a idade e responsabilidades econômicas e familiares. Neste sentido, Sanders e Lushington ${ }^{13}$ compararam 202 estudantes de Medicina Dentária em termos de sua experiência de 
estresse. Por existir um elevado número de estudantes estrangeiros em cada ano do curso, estes tiveram de ser examinados separadamente, considerando as diferenças culturais/étnicas. Já Abramovitch et al. ${ }^{14}$ desenvolveram um instrumento específico para o estudo do estresse dos estudantes de Medicina americanos que estudavam em Israel. Mais ainda, sugerem a realização de um estudo comparativo entre a experiência de estudantes americanos e outros estudantes estrangeiros que efetuam seus estudos noutros países.

O presente estudo pretende contribuir para o desenvolvimento de metodologias de avaliação dos estressores acadêmicos dos estudantes de Medicina em Portugal, por meio do desenvolvimento de um inventário que permita identificar as principais fontes de estresse acadêmico dos estudantes da FMUP e a intensidade com que são experienciadas.

\section{METODOLOGIA}

Na construção do IFSAM, incluíram-se duas fases: uma de desenvolvimento de itens e outra para o teste empírico da fidelidade e validade de instrumentos.

$\mathrm{Na}$ fase inicial, procedeu-se a um trabalho de natureza exploratória, para a construção dos itens do inventário. Tornou-se, então, prioritário conhecer a opinião dos estudantes da FMUP, na perspectiva de estudantes, tendo-se utilizado uma entrevista não estruturada. Assim, e por meio de uma metodologia qualitativa, pretendeu-se obter informação sobre a experiência do estresse acadêmico destes estudantes e conhecer a perspectiva destes sobres quais eram as situações indutoras de estresse mais importantes no curso de Medicina (o procedimento se assemelha ao utilizado nos estudos sobre estresse nos profissionais de saúde ${ }^{15}$ nos psicólogos ${ }^{16}$ e nos docentes ${ }^{17}$ ).

O trabalho de campo decorreu em fevereiro e março de 2004. Estabeleceu-se um primeiro contato com alguns estudantes da Associação de Estudantes da FMUP de forma a apresentar a investigação, seus objetivos e solicitar apoio para a realização das entrevistas. O feedback foi imediato, traduzindo-se num processo de colaboração positiva. As entrevistas, realizadas em grupo (entre 2-6 estudantes), tiveram lugar no Gabinete de Educação Médica, em horários disponibilizados pelos estudantes. Foram entrevistados 80 estudantes dos seis anos da licenciatura (Tabela 1).

A entrevista semiestruturada teve por base uma pergunta aberta - "Enquanto estudantes de Medicina da FMUP, quais as situações que vos causam estresse - isto é, que vos provocam tensão e mal-estar?" -, desencadeando-se uma resposta-debate que durou entre 30 e 90 minutos. As entrevistas foram gravadas, tendo sido anotados os aspectos mais relevantes. Foram efe-
TABELA 1

Síntese do número de entrevistas realizadas para a construção do IFSAM

\begin{tabular}{cccc}
\hline Ano da icenciatura & $\mathbf{n}$ - Feminino & $\mathbf{n}-$ Masculino & Total \\
\hline $1^{\text {o }}$ & 6 & 5 & 11 \\
$2^{\mathrm{o}}$ & 9 & 8 & 17 \\
$3^{\mathrm{o}}$ & 7 & 8 & 15 \\
$4^{\mathrm{o}}$ & 5 & 7 & 12 \\
$5^{\mathrm{o}}$ & 6 & 7 & 13 \\
$6^{\mathrm{o}}$ & 5 & 7 & 12 \\
Total $^{\mathrm{o}}$ & 38 & 42 & 80 \\
\hline
\end{tabular}

tuadas algumas intervenções de forma a esclarecer eventuais dúvidas. As entrevistas foram posteriormente transcritas e procedeu-se à análise qualitativa dos dados (análise de conteúdo), recorrendo-se a um processo de categorização, isto é, uma operação de classificação dos elementos constitutivos dos relatos dos estudantes por classes, as quais reúnem "unidades de registo sob um título genérico"18, de forma a conseguir-se reunir pontos comuns e elucidar os significados atribuídos ${ }^{19}$ às situações de estresse descritas. Quando as fontes de estresse estavam todas inventariadas, foi pedido a dois estudantes de cada ano do curso para analisar a lista, de forma a garantir sua fidelidade e objetividade. A análise de conteúdo resultou numa lista de 31 itens de fontes de estresse acadêmico.

Em termos do formato de resposta, usou-se o mesmo método de McIntyre e colaboradores ${ }^{15}$ no questionário de fontes de estresse profissional. Assim, as fontes de estresse foram convertidas numa escala do tipo Likert, solicitando-se, numa primeira fase, a indicação da intensidade dessa fonte: (1) muito pouco estresse; (2) pouco estresse; (3) estresse médio; (4) muito estresse; (5) estresse extremo; e (NA) não se aplica. Num segundo momento, é pedida a ordenação decrescente das fontes de estresse de forma a se obter um ranking das mesmas.

\section{Validação empírica}

O estudo de validação do IFSAM foi realizado com uma amostra de 251 estudantes dos seis anos do curso (160 do sexo feminino e 91 do sexo masculino). A seleção dos participantes ocorreu nos Serviços Acadêmicos da FMUP. Recorremos ao método da amostragem probabilística, a amostragem aleatória estratificada proporcional. Da totalidade da amostra, 71\% ( $\mathrm{n}=178)$ frequentam um dos anos do ciclo básico $\left(1^{\circ}, 2^{\circ}\right.$ ou $\left.3^{\circ}\right)$ e $29,1 \%(\mathrm{n}=73)$ frequentam um dos anos do ciclo clínico $\left(4^{\circ}\right.$, 
TABELA 2

Consistência interna do IFSAM $(n=251)$

\begin{tabular}{|c|c|c|c|}
\hline & Itens & \multirow{2}{*}{$\begin{array}{c}\begin{array}{c}\text { Correlação com o } \\
\text { total da escala }\end{array} \\
.439\end{array}$} & \multirow{2}{*}{$\begin{array}{c}\text { Alpha } \\
.874\end{array}$} \\
\hline 1 & Preocupação com o sucesso acadêmico & & \\
\hline 2 & Carga horária elevada do curso & .489 & .872 \\
\hline 3 & Transição para a universidade & .251 & .878 \\
\hline 4 & Competição exagerada entre os colegas do curso & .354 & .875 \\
\hline 5 & Número de horas de sono insuficientes & .399 & .874 \\
\hline 6 & Deslocação da residência de origem & .347 & .876 \\
\hline 7 & Acompanhamento regular das matérias & .389 & .875 \\
\hline 8 & Incapacidade de responder às questões dos doentes & .265 & .877 \\
\hline 9 & Dificuldade na gestão de tempo & .419 & .874 \\
\hline 10 & Incertezas quanto ao futuro do Internato Geral & .228 & .878 \\
\hline 11 & Exigências da disciplina de Anatomia & .370 & .875 \\
\hline 12 & Responsabilidades éticas/humanas do médico & .356 & .875 \\
\hline 13 & Dedicação exigida pelo curso & .517 & .872 \\
\hline 14 & Ambiente físico da FMUP & .251 & .878 \\
\hline 15 & Ambiguidade nos critérios de correcção & .311 & .876 \\
\hline 16 & Dificuldades no método de estudo & .460 & .873 \\
\hline 17 & Relação com os professores & .441 & .873 \\
\hline 18 & Volume elevado de matérias para estudar & .537 & .871 \\
\hline 19 & Expectativas familiares elevadas & .314 & .876 \\
\hline 20 & Dificuldade em manter uma alimentação equilibrada & .394 & .875 \\
\hline 21 & Ritmo das avaliações/exames & .515 & .872 \\
\hline 22 & Expectativas sociais em relação ao estatuto & .460 & .873 \\
\hline 23 & Contato directo com o doente & .271 & .877 \\
\hline 24 & Ensino/aprendizagem focados demasiadamente na memorização & .500 & .872 \\
\hline 25 & Falta de tempo para atividades de lazer & .540 & .871 \\
\hline 26 & Adaptação às exigências acadêmicas da universidade & .492 & .872 \\
\hline 27 & Relações com os profissionais nos serviços do hospital & .361 & .875 \\
\hline 28 & Falta de tempo para os amigos e/ou família & .586 & .870 \\
\hline 29 & Pressão dos colegas para o sucesso & .418 & .874 \\
\hline 30 & Pouca preparação prática & .339 & .876 \\
\hline 31 & Sistema de avaliação & .551 & .871 \\
\hline & $\begin{array}{l}\text { dia de Cronbach } \\
\text { dia }\end{array}$ & & \\
\hline
\end{tabular}

$5^{\circ}$ ou $\left.6^{\circ}\right)$. Os estudantes receberam um pacote de investigação que incluía consentimento informado, questionário sociodemográfico e questionários de avaliação do estresse, saúde geral, estilos de vida e o IFSAM.

Após a recepção dos questionários, a investigadora responsável procedeu à cotação dos itens do IFSAM, de acordo com os critérios predefinidos. O tratamento estatístico dos dados teve suporte informático no programa Statistical Package for Social Sciences - SPSS, versão 14.0 para Windows.
Procedeu-se ao estudo das características psicométricas do instrumento em termos da análise de fidelidade (consistência interna) e da validade de constructo.

Considerando a importância de avaliar a sensibilidade e fidelidade dos resultados, garantindo "o grau de uniformidade e de coerência existente entre as respostas dos sujeitos a cada um dos itens que compõem a prova" (Almeida \& Freire, 2000), foi realizada uma análise da consistência interna dos itens (Tabela 2). Foi determinado o coeficiente alpha de Cronbach 
e encontrado um coeficiente de fidelidade para o IFSAM total de.88, revelando uma consistência interna muito boa. Não foi necessário excluir nenhum item, uma vez que nenhum deles, se eliminado, aumentaria o valor do alpha. As correlações totais destes itens com o total da escala variavam entre. 25 e.59.

\section{TABELA 3}

IFSAM - Validade de constructo: resultados da análise fatorial dos componentes principais com rotação varimax

$$
\text { ( } \mathrm{n}=\mathbf{2 5 1})
$$

\begin{tabular}{cccc}
\hline Fator & $\begin{array}{c}\text { Carga } \\
\text { fatorial }\end{array}$ & $\begin{array}{c}\text { \% Variância } \\
\text { explicada }\end{array}$ & $\begin{array}{c}\text { \% Variância } \\
\text { cumulativa }\end{array}$ \\
\hline 1: Exigências do curso & & & \\
\hline 18 & .727 & & \\
21 & .639 & & 16,9 \\
16 & .634 & & \\
7 & .633 & & \\
11 & .575 & & \\
24 & .556 & & \\
13 & .555 & & \\
9 & .553 & & \\
1 & .505 & & \\
\hline
\end{tabular}

2: Exigências humanas

$\begin{array}{llll}12 & .677 & & \\ 23 & .618 & 11,9 & 28,7 \\ 22 & .600 & & \\ 17 & .521 & & \end{array}$

3: Estilo de vida

\begin{tabular}{cccc}
\hline 5 & .706 & & \\
28 & .610 & & \\
20 & .604 & 10,2 & 39,0 \\
25 & .556 & & \\
2 & .524 & & \\
\hline
\end{tabular}

4: Competição

\begin{tabular}{cccc}
\hline 29 & .661 & 8 & 41 \\
4 & .634 & 8,1 & 47,1 \\
\hline
\end{tabular}

5: Adaptação

\begin{tabular}{crcc}
\hline 3 & .730 & 7,7 & 54,8 \\
26 & .613 & 7,7 & \\
\hline \% Total de variância explicada & & 54,8
\end{tabular}

A validade de constructo da escala foi avaliada por meio de procedimentos de análise fatorial e coeficientes de correlações de Pearson. Assim, os 31 itens foram submetidos a uma análise fatorial do tipo exploratório, da qual se obtiveram, após rotação varimax dos eixos, nove fatores. Todavia, alguns desses fatores eram insignificantes, quer em termos da variância explicada, quer de sua interpretabilidade. Procedeu-se, então, à exploração de soluções fatoriais do menor número de fatores $(5,6,7$ e 8$)$ para obter uma solução mais adequada. A solução de cinco fatores pareceu-nos a mais adequada em termos da variância explicada $(54,8 \%)$ e da representatividade do constructo. Foram considerados os itens com a carga fatorial $>.50$. Obtivemos, assim, uma estrutura fatorial coerente com os resultados de outros estudos com estudantes de Medicina realizados noutros países (Tabelas 3 e 4). O primeiro fator - "exigências do curso" -, com 16,9\% da variância explicada, contempla todos os itens (nove) que se referem à elevada dedicação exigida pelo curso de Medicina, o número de horas e o volume de matéria para estudar. O segundo fator, constituído por quatro itens - "exigências humanas" -, diz respeito às relações com os doentes e/ou os profissionais de saúde com quem têm de lidar no decorrer da licenciatura, explicando $11,9 \%$ da variância. O terceiro fator - "estilos de vida" -, integrando cinco itens, refere-se às dificuldades em gerir uma alimentação saudável, à prática de exercício físico e ao sono (10,2\% de variância explicada). Os últimos dois fatores - competição e adaptação, com dois itens cada - englobam aspectos relacionados com a pressão dos colegas em torno do sucesso acadêmico, a competição entre os colegas e as dificuldades inerentes à adaptação ao curso de Medicina e ao ensino superior, respectivamente, explicando $8,1 \%$ e 7,7\% da variância.

\section{TABELA 4}

IFSAM - Estatísticas descritivas, consistência interna e coeficientes alfa das subescalas do IFSAM $(n=251)$

\begin{tabular}{lcccc}
\hline \multicolumn{1}{c}{ Fatores } & No itens & M & DP & Alfa \\
\hline I - Exigências do curso & 9 & 31.243 & 5.9539 & .8214 \\
II - Exigências humanas & 4 & 9.502 & 2.737 & .6573 \\
III - Estilo de vida & 5 & 14.892 & 3.866 & .7417 \\
IV - Competição & 2 & 4.326 & 1.686 & .6166 \\
V - Adaptação & 2 & 6.083 & 1.851 & .6840 \\
\hline
\end{tabular}

Com base nos fatores encontrados, constituíram-se as correspondentes subescalas (derivação empírica). Em seguida, foi estimada a consistência interna das escalas, cujos resultados são apresentados na Tabela 4 . Os resultados da análise de fidelidade revelam coeficientes de alfa que variam entre.62 e.82, indicando uma consistência interna razoável a boa.

Com vista ao estudo da relação entre as subescalas do IFSAM e a escala total, foram determinados os coeficientes de correlação de Pearson. Na Tabela 5, observa-se que, quando são correlacionadas as cinco fontes de estresse entre si, verifica-se que a correlação mais forte se verificou entre as "exigências do curso" e o "estilo de vida" (.55). As correlações entre as subescalas são de baixas a moderadas, confirmando a especificidade destas. 
TABELA 5

Coeficientes de correlação de Pearson para as subescalas do IFSAM (n = 251)

\begin{tabular}{|c|c|c|c|c|c|}
\hline & Exigências do curso & $\begin{array}{c}\text { Estilo } \\
\text { de vida }\end{array}$ & Exigências humanas & Competição & Adaptação \\
\hline Exigências do curso & & $.548^{* *}$ & $.261^{* *}$ & $.275^{* *}$ & $.367^{* *}$ \\
\hline Estilo de vida & $.548^{* *}$ & & $.326^{* *}$ & $.363^{* *}$ & $.253^{* *}$ \\
\hline Exigências humanas & $.261^{* *}$ & $.326^{* *}$ & & $.317^{* *}$ & $.300^{* *}$ \\
\hline Competição & $.275^{* *}$ & $.363^{* *}$ & $.317^{* *}$ & & .092 \\
\hline Adaptação & $.367^{* *}$ & $.253^{* *}$ & $.300^{* *}$ & .092 & \\
\hline
\end{tabular}

Nota: ${ }^{*} \mathrm{p}<.05 \quad * * \mathrm{p}<.01 \quad * * * \mathrm{p}<.001$

\section{CONCLUSÕES}

O estresse na formação médica inicial e suas possíveis consequências parecem, por vezes, ser negligenciados no contexto educacional, já que poucas faculdades de Medicina contam com um serviço específico de apoio aos estudantes. É certo que a maioria dos estudantes de Medicina lida com sucesso com as exigências de suas vidas, mas também é um fato que cerca de $30 \%$ destes desenvolvem problemas psicológicos ${ }^{9}$. Na opinião de Adams ${ }^{3}$, Bligh ${ }^{20}$ e Cataldo Neto e colaboradores ${ }^{21}$, as escolas médicas precisam melhorar o padrão de ensino (mesmo no nível da educação para a saúde) que ministram, adaptando-se e interagindo melhor com o desenvolvimento do seu objeto de ensino: o estudante de Medicina, com suas características psicossociais e o estresse a que é submetido.

Em termos globais, vários estudos sugerem que, em termos de intervenção, as estratégias devem ser multifacetadas, dirigidas quer ao indivíduo (estudante), quer ao curso, englobando também outros aspectos do contexto acadêmico, como ambiente físico e atividades extracurriculares. Deste modo, parece fundamental uma avaliação precisa dos estressores ou principais dificuldades destes estudantes, de modo a desenvolver programas de intervenção e de prevenção mais apropriados aos estudantes de Medicina e implementar um currículo médico mais ajustado ${ }^{22}$.

Em conclusão, após analisadas as propriedades psicométricas do IFSAM, verifica-se que este apresenta boa fidelidade e validade de constructo. Neste sentido, podemos considerar o IFSAM um instrumento útil na avaliação das fontes e intensidade de estresse acadêmico do curso de Medicina na Faculdade de Medicina do Porto. Será necessário, contudo, aplicar este instrumento em outros cursos de Medicina do país, para verificar sua aplicabilidade mais generalizada, uma vez que a literatura nos chama a atenção para a necessidade de considerar as diferenças culturais ${ }^{13,14,23}$ e até as do próprio currículo médico ${ }^{24,25}$, o que permitiria, posteriormente, estudos compa- rativos dos fatores, níveis e respostas de estresse dos estudantes de Medicina.

\section{REFERÊNCIAS}

1. Ravindranauth D. Estresse in the medical profession: an evaluation of pre-medical students, medical students and doctors. Experimental Sciences. 2000 [online]. [acesso em: ]. Disponível em: http://socrates.berkeley.edu/ es196/ projects/2000final/ravindranath.pdf

2. Peters D. Self-care: estresse and the practitioner. In: A. Watkins (ed.). Mind and body medicine: a clinician's guide to Psychoneuroimmunology. Churchill Livingstone, San Francisco:[s.n]; 1997.

3. Adams J. Straining to describe and tackle estresse in medical students. Medical Education. 2004; 38:463-464.

4. Bhat K. Personal Comunication. In: D. Ravindranauth (ed.). Estresse in the medical profession: an evaluation of pre-medical students, medical students and doctors. Experimental Sciences. 1999. [online]. [acesso em: ]. Disponível em: http://socrates.berkeley.edu/ es196/projects/ 2000final/ravindranath.pdf

5. Vitaliano P, Maiuro R, Russo J, Becker J. Raw versus relative scores in the assessment of coping strategies. In: Abramovitch $\mathrm{H}$, Schreier A, Koren N (eds.). American Medical Students in Israel: estresse and coping: a follow-up study. Medical Education. 1987; 34: 890-896.

6. Knight J. Doctor-to-be. Coping with the trials and triumphs of Medical School. Appleton-Century Crofts, NY:[s.n.]; 1981.

7. Miller P. The first year at medical school: some findings and student perceptions. British Journal of Psychiatry. 1994;159:199-207.

8. Wolf T, Von Almen T, Faucett J, Randall H, Franklin F. Psychosocial changes during the first year of medical school. Medical Education. 1991;25:174-181. 
9. Shaw D, Wedding D, Zeldow P, Diehl N. Special problems of medical students. [S.1.:s.n.]; 2002.

10. Toews C, Lockyer J, Dobson D, Simpson E, Brownell A, Brenneis $\mathrm{F}$ et al. Analysis of estresse levels among medical students, residents and graduate students at four Canadian schools of medicine. Acad. med. 1997; 72(11):997-1002.

11. Coles C. Introduction: medicine and estresse. Medical Education. 1994;28:3-4.

12. Lazarus R, Folkman S. Estresse, appraisal and coping. NY: Springler; 1991.

13. Sanders A, Lushington K. Effect of perceived estresse on student performance in Dental School. Journal of Dental Education. 2002; 66(1).

14. Abramovitch H, Schreier A, Koren N. American medical students in Israel: estresse and coping a follow-up study. Medical Education. 2000;34:890-896.

15. McIntyre, McIntyre \& Silvério (2000). Work Estresse and job satisfaction in Portuguese health professionals. Occupational Health Psychology: Europe. 2000:105 -111.

16. Gomes AR. Estresse e "burnout" nos profissionais de Psicologia. Braga; 1998. Mestrado [Dissertação] - Universidade do Minho.

17. Mota-Cardoso R, Araújo A, Carreira R, Gonçalves G, Ramos M. O estresse nos professores portugueses. Estudo IPSSO 2000. Caramulo, Portugal: Instituto de Prevenção do Estresse e Saúde Ocupacional; 2000.

18. Bardin L. Análise de Conteúdo. Lisboa: Edições 70; 1977.

19. Patton MQ. Qualitative Research \& Evaluation Methods. California: Sage Publications; 2002

20. Bligh J. More medical students, more estresse in the medical education system. Medical Education. 2004; 38(5):460462.

21. Cataldo Neto A, Cavalet D, Bruxel DM, Kappes DS, Silva DOF. O estudante de medicina e o estresse académico. Rev. med PUCRS. 1998; 8(1): 6-12.

22. Johnston M, Johnston D. Assessment and Measurement issues. Health Psychology. 1998; 8:114-135.
23. Moffat K, McConnachie A, Ross S, Morrison J. First year medical student estresse and coping in a problembased learning medical curriculum. Medical Education. 2004;38(5):482-491.

24. Kiessling C, Schubert B, Scheffner D, Burger W. First year medical students' perceptions of estresse and support: a comparison between reformed and traditional track curricula. Medical Education. 2004;38:504-509.

25. Moffat K, Ross S, Morrison J. A questionnaire to examine medical student estresse and coping development, content and administration. Dublin: ASME Scientific Meeting; 2001.

\section{CONTRIBUIÇÃO DOS AUTORES}

$\mathrm{O}$ artigo resultou de uma pesquisa realizada por Elizabete Loureiro, baseando-se num instrumento já validado em estudos prévios por Teresa McIntyre, que supervisionou todos os procedimentos do estudo juntamente com Rui Mota Cardoso. Maria Amélia Ferreira participou ativamente na realização das entrevistas aos estudantes de Medicina e contribuiu, junto de Elizabete Loureiro, para a análise e discussão dos resultados. Elizabete Loureiro redigiu o artigo final, tendo sido previamente delineado entre todos os autores que também procederam às correções finais antes de sua submissão à Revista Brasileira de Educação Médica.

\section{CONFLITO DE INTERESSES}

Declarou não haver

\section{ENDEREÇO PARA CORRESPONDÊNCIA}

Elizabete Maria Ferraz Loureiro Centro de Educação Médica - Piso 6 Faculdade de Medicina da Universidade do Porto Alameda Hernâni Monteiro CEP.: 4200-319 - Porto - Portugal E-mail: gempsico@med.up.pt 